\title{
Propuesta para la Enseñanza de la Ingeniería: Un Espacio Curricular Creado desde la Investigación- Acción
}

\author{
María del C. Castells, Alicia N. Arese, Enrique D. Albizzati y Germán H. Rossetti \\ Universidad Nacional del Litoral, Facultad de Ingeniería Química, Santiago del Estero 2829, \\ (3000) Santa Fe-Argentina (e-mail: mariqui_c@yahoo.com.ar, aarese@fiq.unl.edu.ar, \\ albizati@fiq.unl.edu.ar, groseti@fiq.unl.edu.ar)
}

\begin{abstract}
Resumen
Se expone el proceso de construcción de una materia optativa para la enseñanza de Ingeniería Química, basada en la investigación-acción. En este contexto, desde una perspectiva interpretativa, los docentes investigadores modificaron el enfoque metodológico de sus clases y crearon estrategias pertinentes para distintas situaciones de enseñanza en el Área de las Operaciones Unitarias. Dichas estrategias convergieron en un espacio curricular integrador de conocimientos de la Ingeniería. Como ejemplo de aplicación de la estrategia propuesta, se desarrolló un caso en el tema de la energía solar. La experiencia de investigación-acción confirmó ser un espacio válido para la transformación y creación de estrategias flexibles, que articulen en la enseñanza de las ciencias y de las tecnologías, cuestiones sociales, frecuentemente soslayadas en Carreras de Ingeniería.
\end{abstract}

Palabras clave: investigación-acción, enseñanza de la ingeniería, estrategias de enseñanza, energía solar

\section{Proposal for the Teaching of Engineering: A Curricular Space Created from Research-Action}

\begin{abstract}
The design of an optional course in chemical engineering based on the concept of research-action is shown here. In this context, from an interpretative point of view, instructor-researchers produced changes in the methodological approach of their teaching and created relevant strategies for different teaching situations in the area of Unit Operations. These strategies converged into an integrating curricular programme of expertise in the area of Engineering. As an example of the method proposed, a study case in the area of solar energy was developed. The research-action experience has proved to be a valid space for the transformation and creation of flexible strategies that articulate, in the teaching of sciences and technologies, social matters usually not considered in the Engineering Careers.
\end{abstract}

Keywords: research-action, engineering teaching, teaching strategies, solar energy 


\section{INTRODUCCIÓN}

Es frecuente que los docentes dedicados a la enseñanza de la Ingeniería, por su formación, instrumenten técnicas de enseñanza sin analizar los principios de procedimiento que las recorren (Arese et al., 2001). Al mismo tiempo, por una larga tradición epistemológica de corte positivista en el campo, es habitual encontrar planes de estudio con estructuras disciplinares rígidas que poseen escasas relaciones multidisciplinares, así como incipientes articulaciones con problemáticas sociopolíticas y ambientales (Castells et al., 2005). En todo caso, lo que es dable observar son yuxtaposiciones de materias que, con el afán de actualizar los planes de estudio, se agregan a estructuras tradicionales.

Ante esta problemática, un grupo Ingenieros Químicos, docentes e investigadores del Área de Operaciones Unitarias, junto a una magíster en educación, en la Facultad de Ingeniería Química de la Universidad Nacional del Litoral de la ciudad de Santa Fe, Argentina, decidieron implicarse en un proyecto de investigación - acción (Stenhouse, 1991; Elliot, 1993) a los fines de transformar criterios prácticos y con ello, generar nuevas estrategias de enseñanza. En ese sentido, se recuperaron experiencias de investigación acción llevadas adelante en diversos ámbitos educacionales (Maciel de Olivera, 2003), reconociendo la pertinencia de esta perspectiva para pensar y concretar la enseñanza.

Tomada la noción de estrategia como concepto básico, se revisaron diferentes concepciones de la misma. Una línea de investigación con fuerte influencia de la psicología educativa constructivista (Monereo, 1998; Pozo y Monereo, 2002), remite a una definición de estrategias de enseñar y de aprender como procesos situados, conscientes e intencionales, consistentes en tomar decisiones en pos del logro de objetivos de aprendizaje o de enseñanza. Se analizó que lo que subyace a esta concepción es considerar estrategias y prácticas escolares en tanto voluntarias y deliberativas en orden a fines establecidos racionalmente.

Sin embargo, desde otras influencias disciplinares, tales como la sociología y la filosofía, la noción de estrategia, situada en el marco de las prácticas sociales amplias, ha sido definida por los habitus (Bourdieu, 1991) o principios estructurantes de las prácticas que no son, según Pierre Bourdieu, ni totalmente conscientes ni totalmente inconscientes; su conformación depende de una relación práctica con el contexto y con experiencias sociales (en el caso de la enseñanza, con las experiencias de haber sido alumno, haber pertenecido o pertenecer a determinados planes de estudio etc., realizar determinadas prácticas docentes, interactuar con diferentes prácticas de investigación o de trabajo profesional en industrias). A pesar de ello, estas acciones no son errantes, poseen la orientación sensata que le da el estar, el entender o el pertenecer a una especie de campo de juego, jugando un juego que adhiere a principios prácticos -para actuar-, sin necesariamente cumplir racionalmente con reglamentos. Las prácticas, prevén el juego, se adelantan, generan estrategias para el juego y su sensatez proviene, no de una adhesión consciente a reglas, sino de un vínculo inteligible con las propias condiciones y posibilidades de su realización. Así, las prácticas muchas veces son inciertas, vagas, indeterminadas y, a pesar de ello tienen su propia coherencia, nunca posible de representarse desde una práctica y una lógica teóricas que no aspiren a ser conscientes de sus propias características y condiciones de producción.

Enmarcando teóricamente el proyecto en esta última perspectiva, en el desarrollo de la investigación- acción, la mayor tarea estuvo concentrada en interpretar para poder transformar los modos en que los principios de procedimientos se hacían presentes en las prácticas de los ingenieros dedicados a la enseñanza. En este sentido, se revisaron los conceptos tácitos de las estrategias vigentes y se reformularon, para arribar a la elaboración y ensayo de nuevas estrategias para enseñar. Fue en este proceso donde el equipo puso en cuestión sus significaciones de conocimiento, de ciencia, de enseñanza, de aprendizaje, las representaciones del oficio docente y del oficio alumno, la relación tiempo-contenidos, la relación teoría experimentación. Las sucesivas estrategias que se implementaron estuvieron guiadas por criterios que intentaron romper con racionalidades lineales (Najmanovich, 2005), proponer otros "usos" de 
las teorías, otras relaciones entre ésta, la experimentación y los problemas. Ello promovió nuevas posiciones de los sujetos en el proceso de enseñanza y de aprendizaje.

En la etapa final de proyecto de investigación se capitalizaron experiencias del equipo que se volcaron en una propuesta de enseñanza que excede el marco de las asignaturas existentes. Conscientes de que en determinadas prácticas de enseñanza de la ingeniería, permanecen aún espacios de simplificación y de disyunción del conocimiento (Morin, 1988), se plantearon algunos criterios que inciden en la estructuración final de una materia optativa integrada (Saraiva y otros, 2007; Brennecke y Stadtherr, 2002). De esta manera, se conformó un espacio de convergencia abierto y articulador de criterios epistemológicos, sociales, políticos y profesionales, que incluya temáticas referidas a innovaciones tecnológicas que, desarrolladas a término, revisen en forma constante su pertinencia cultural y su legitimidad epistemológica (Chevallard, 1997). Así, se ha considerado de relevancia la incorporación de las prácticas de investigadores, de profesionales del área productiva, de diferentes actores sociales, de científicos sociales, junto con los docentesinvestigadores del área.

\section{METODOLOGÍA}

El proceso de diseño de nuevas estrategias de enseñanza en el área de Operaciones Unitarias, hasta llegar a la construcción del nuevo espacio curricular, se realizó en el marco metodológico de la investigación- acción, asumiéndose un enfoque eminentemente interpretativo (Elliot, 1993). Con lo cual, las conclusiones del proyecto lejos de considerarse generalizables, se concibieron como la producción de un conocimiento sustantivo, es decir, pertinente para la situación indagada.

El camino trazado, a partir del problema de la investigación y de su conceptualización temática, fue el propuesto por esta metodología elegida: identificación del problema, plan de acción, implementación, revisión de la implementación, nueva identificación del problema, nuevo plan de acción, revisión (Elliot, 1993). El grupo de investigación, con una observadora participante, construyó diversos instrumentos de recogida de la información, tales como registros, observaciones de clases, entrevistas en profundidad, todos, tendientes a hacer emerger los principios tácitos de las estrategias de enseñanza y los significados anudados a ellos, vigentes en ese momento, para que, a posteriori de sus análisis y confrontación con nuevos conocimientos, pudieran implementarse otros principios que dieran lugar a otras estrategias.

Luego de la discusión de los significados de los conceptos mencionados en el apartado anterior, se diseñaron estrategias que se denominaron: "Trabajo Práctico Especial" (Albizzati et al. 1998) y "Resolución de problemas teórico-prácticos" (Arese et al., 2000), que se evaluaron y se rediseñaron a la luz de los resultados obtenidos. Para cada uno de los momentos se implementó un proceso de evaluación que, en este proyecto de investigación no tuvieron una intencionalidad confirmatoria de los cambios acontecidos y de la efectividad de las estrategias implementadas. Tuvieron, sobre todo, una intencionalidad creativa, en el sentido de promover la apertura de nuevos problemas y de nuevas propuestas.

En este sentido, se hace necesario mencionar que los debates en torno a los paradigmas contemporáneos de la Ingeniería Química estuvieron presentes en los intercambios entre los miembros del equipo.

En efecto, desde finales del siglo XX, varios autores sostuvieron que la industria de los procesos químicos se enfrentaba a grandes retos. Entre ellos, Tapias García (1999), señalaba: "el desarrollo de procesos más aceptables socialmente...; el desarrollo de procesos que utilicen nuevas materias primas y de más baja calidad...; el diseño de plantas con tamaños económicos mínimos más pequeños, costes de construcción más bajos, equipos estándares y modulares y plantas flexibles multiproducto; el desarrollo de nuevos procesos con innovaciones tecnológicas como las que se vienen dando con la hibridación de procesos y operaciones unitarias...". Por otra parte, a principios del tercer milenio, se mencionaron como desafíos con los cuales se confrontarían las industrias químicas en el marco de un mercado y una competitividad globalizada, la creación de procesos innovativos no contaminantes, libres de defectos y perfectamente seguros para la producción de commodities y productos intermedios, así como el desplazamiento desde la 
química tradicional hacia nuevas especialidades donde la relevancia esté puesta en la síntesis de productos requeridos por el consumidor, con determinadas propiedades de uso que resulten confiables y no alteren demasiado el ambiente. Además, se vaticinaba para la disciplina la necesidad de contar con una visión global sobre el desarrollo y diseño de productos (Charpentier, 2002).

En la última década cobró fuerza dentro de la Ingeniería Química el concepto de "ingeniería de los productos químicos". Según Costa et al. (2006), dicho concepto designa el marco de conocimientos, enfoques, metodologías y herramientas empleadas para analizar, desarrollar y producir un amplio rango de productos químicos con propiedades de uso final consideradas valiosas por el consumidor.

Así, por estos días las modernas industrias de procesos químicos demandan el desarrollo de nuevos conceptos y un cambio en la forma de utilización de las herramientas de la Ingeniería Química, perfilándose el 3er. Paradigma de la disciplina alrededor del concepto de "ingeniería de los productos químicos" (Costa et al., 2006).

Estos cambios tan radicales en los modos de concebir la ingeniería química implicó al equipo de investigación en la búsqueda de transformaciones de su propuesta de enseñanza. De este modo, como parte del proyecto se generó una instancia de formación de los miembros del equipo que abrió límites disciplinarios y ubicó los contenidos específicos de las Operaciones Unitarias en un marco de debates vinculados a las problemáticas contemporáneas del conocimiento (Morin, 1998; Najmanovich, 2005), del trabajo (Castells, 2000; Bauman, 2007) y del desarrollo sustentable (Osorio, 2004; Pereira, 1999).

Los desafíos de la sociedad, de la tecnología y de la industria contemporáneas contribuyeron al convencimiento de que es necesario optar, desde la formación universitaria de grado, por una perspectiva de conocimiento cuyos principios permitan abordajes diferentes a los establecidos por los paradigmas clásicos, aproximando la enseñanza de la ingeniería química al 3er. paradigma (Wintermantel, 1999; Charpentier, 2002; Costa et., 2006).

\section{RESULTADOS Y DISCUSIÓN}

El espacio curricular optativo se ha pensado en un diseño cíclico (Camilloni etal., 2002), recursivo y abierto a su relación con el entorno (Morin, 1998; Najmanovich, 2005). Por ello, si bien se recuperaron los ejes que hacen a la estructura sintáctica y semántica del área de los Fenómenos de Transporte y las Operaciones Unitarias, los mismos se vinculan con diferentes problemáticas y temas de relevancia epistemológica y cultural, a partir de lo cual, la estructura disciplinar cobra diversas especificidades y obliga a reformulaciones continuas. Se piensa que esta materia sea un ámbito flexible donde se desprendan y se especifiquen temas puntuales que convoquen miradas y prácticas multidisciplinares, pertinentes con la formación actual del profesional de la ingeniería, teniendo en cuenta que su actividad no se circunscribe solamente a la esfera técnica, sino que transita desde lo técnico a lo social. (Costa et al., 2006; Gutiérrez Martin, 2007; Saraiva et al., 2007). Desde aportes multidisciplinares, en la materia optativa, los estudiantes podrán integrar las ecuaciones de balance de materia, energía y movimiento, que en el desarrollo de las asignaturas del área se analizan en forma separada por razones didácticas.

La metodología de enseñanza seleccionada para la materia optativa es la de seminario -proyecto y tiene la intención de promover la participación activa y permanente de los alumnos, sostenida por el debate argumentado. Se pretende que al finalizar el cursado de la materia los estudiantes hayan elaborado un proyecto que genere potencialmente un desarrollo social a partir de la innovación tecnológica. Para su realización será necesario tomar contacto con el estado del arte de la temática elegida así como con situaciones sociales concretas, con las especificaciones industriales y las comerciales, los requisitos legales y éticos para la producción e implementación de lo propuesto. Se hará hincapié, aunque no en una relación de determinación, en el desarrollo tecnológico y su vínculo con las necesidades sociales y características culturales. 
Durante el devenir del proyecto se recorrerán alternativamente algunas de las siguientes etapas: planteo de la problemática social y tecnológico-científica, análisis teórico, diseño y eventualmente construcción del equipo, modelado y simulación, obtención de datos experimentales, mejora de detalles constructivos y de técnicas de medición, análisis de datos, interpretación de resultados, elaboración de conclusiones, presentación oral y escrita, debate.

Cabe aclarar que cada uno de los momentos de la elaboración del proyecto por parte de los alumnos serán instancias para evaluar y reformular la enseñanza y los aprendizajes, así como para proponer nuevas temáticas que reorienten y actualicen este espacio curricular.

Se presenta aquí, desde este marco del desarrollo del proceso de investigación acción, la estructura de dicha materia, sus justificaciones teórico-metodológicas, en torno a un ejemplo desarrollado sobre un caso temático puntual tal como es la energía solar.

\section{Energía solar: a modo de ejemplo. Análisis de un caso particular para la materia optativa}

Es comúnmente aceptado que la energía es un recurso que debe contribuir al desarrollo humano, brindando opciones y oportunidades a los hombres y mujeres de nuestro planeta. Sin embargo, debe considerarse también que la generación y uso de la energía está acompañada de efectos ambientales a nivel local, regional y mundial, que a su vez amenazan el bienestar humano: el empleo intensivo de combustibles fósiles (carbón, petróleo) en los países industrializados, y la consiguiente emisión de gases de efecto invernadero, está provocando el denominado Cambio Climático Global (aumento en la temperatura media de la superficie terrestre). Resulta entonces un desafío introducir tecnologías energéticas que respondan a las pautas establecidas para las energías, en el marco de un desarrollo sustentable. Debe hacerse notar que durante el Foro Solar Milenio 2000, organizado por la Sociedad Internacional de Energía Solar en la ciudad de México, los científicos y técnicos participantes coincidieron en que "no existen obstáculos tecnológicos importantes para la aplicación las energías renovables, y en cambio las principales barreras son políticas, sociales económicas y culturales".

Entre las energías renovables y no contaminantes, la energía solar y las tecnologías aplicables para su transformación, son algunos de los recursos más importantes con que se cuenta hoy para modificar el sistema energético establecido a nivel mundial. Actualmente se emplea la energía solar principalmente como energía térmica a bajas y medias temperaturas y en la obtención de electricidad mediante celdas fotovoltaicas. Además se avanza hacia la aplicación de la energía solar en altas temperaturas, para generar calor en procesos industriales y para la generación de electricidad en ciclos térmicos. Otro campo en el cual la radiación solar tiende a lograr una importante participación en el futuro, es en la degradación de sustancias tóxicas presentes en efluentes industriales o desechos de consumo humano mediante procesos fotoquímicos y fotocatalíticos, convirtiéndolas en productos que no dañen el ambiente.

Por estos motivos enunciados, resulta muy importante la inclusión en las Carreras de Ingeniería de temas relacionados con energías sustentables, y entre ellas la energía solar, donde los estudiantes adquieran los conocimientos básicos relacionados con estas fuentes y puedan realizar trabajos prácticos para estudiar la dinámica y los fenómenos que se presentan en los equipos de uso más extendido (Albizzati, 2000; Albizzati y Rosseti, 2002), así como avanzar en propuestas novedosas acerca del aprovechamiento de esta fuente energética renovable.

En la figura la Figura 1, se presenta un abanico de posibles relaciones en torno al eje seleccionado, como un modo de ejemplificar la estructura básica que tendrá la asignatura optativa.

Durante el desarrollo de la misma y en el marco del seminario se deben introducir los conceptos necesarios para la comprensión de los fenómenos relacionados con la energía solar. Ellos son: el comportamiento de los diversos materiales frente a la radiación solar, especialmente la variación de sus propiedades ópticas a lo largo del espectro, la formulación de ecuaciones de balance térmico, y el análisis de los mecanismos de Conducción, Convección y Radiación en los dispositivos de conversión de energía solar en energía térmica. 


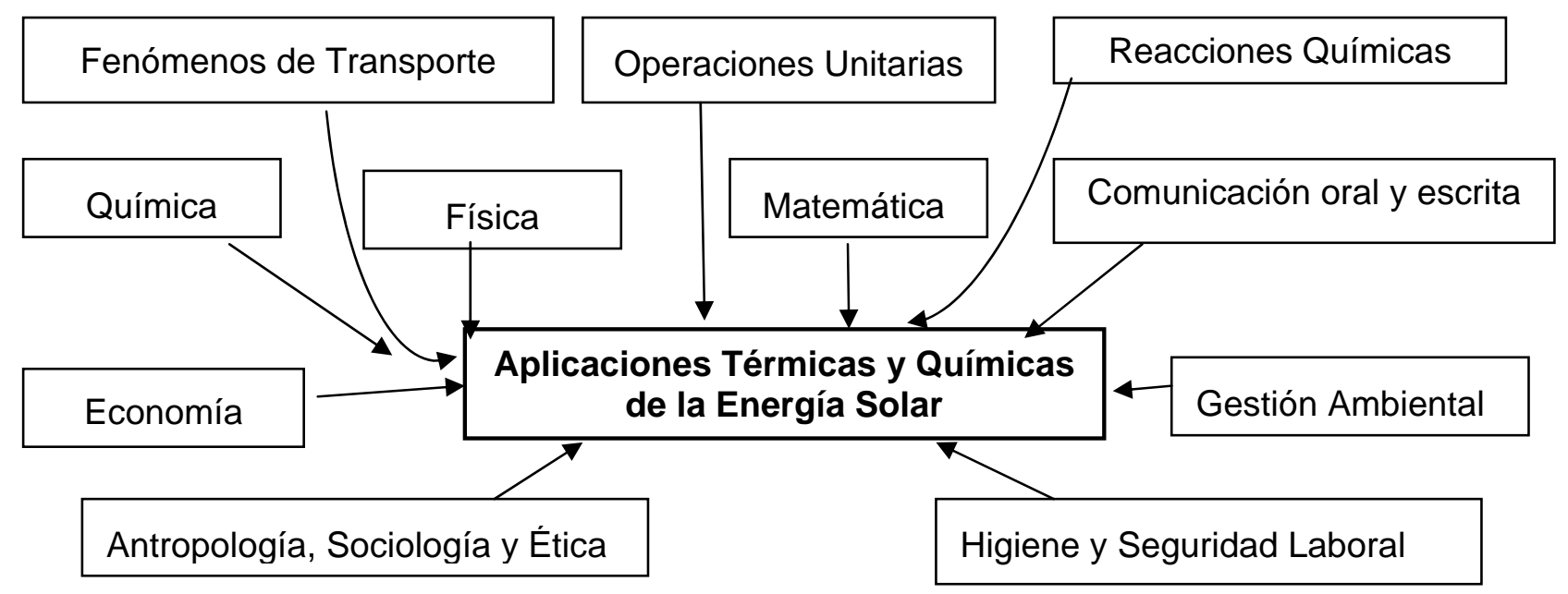

Fig. 1: Estructura básica de la asignatura optativa propuesta

También, y tal como se especificó en la sección anterior en alusión a la estructura cíclica que se propone para la materia optativa, en ella se recuperan los ejes temáticos que atraviesan toda el área de Fenómenos de Transporte y Operaciones, que se especifican en la temática puntual. Los ejes y contenidos de esta asignatura serían móviles en función de los intereses sociales, de los aportes que los docentes investigadores puedan hacer desde su campo de indagación (como un modo de aproximar las tareas de investigación y docencia), de las interpelaciones de los alumnos.

En la Figura 2 se sintetizan los ejes, principios, conceptos y metodología del área, que se retqman en la asignatura propuesta.

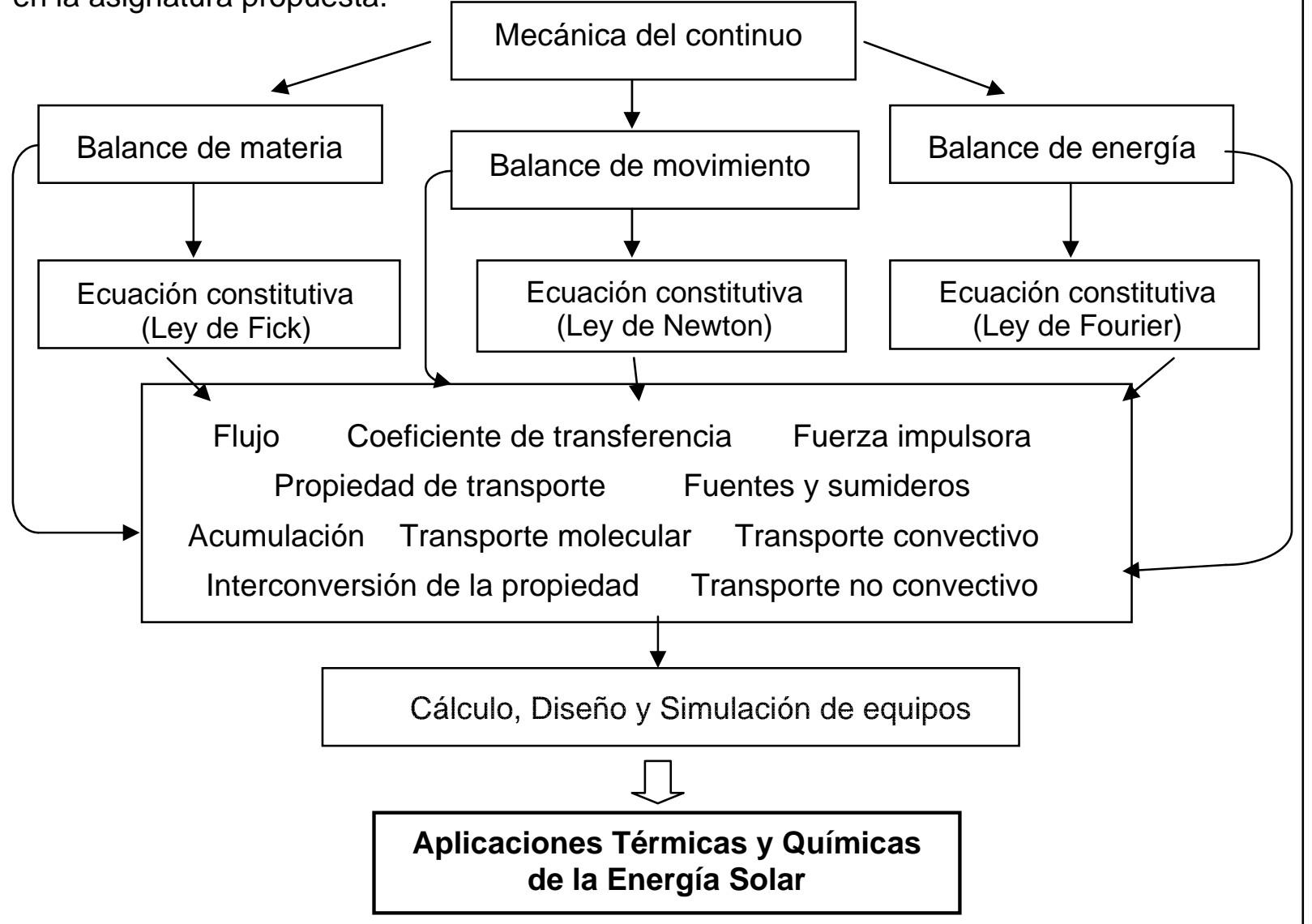

Fig. 2: Ejes, principios, conceptos y metodología del área de Operaciones Unitarias. 


\section{CONCLUSIONES}

Se destaca que la propuesta y el desarrollo de esta asignatura optativa, constituye una concreción sustantiva de transferencia de procesos y de resultados de la investigación a la enseñanza. Esta transferencia se da en un doble aspecto: desde el campo de la investigación educacional llevado adelante por todo este equipo y desde el campo de la investigación en energía solar por parte de algunos de sus miembros.

La asignatura optativa, tal como está planteada ofrece a los alumnos una posibilidad de integrar los aprendizajes curriculares con las futuras actividades profesionales, ya que la metodología retoma toda la experiencia desarrollada por el grupo de trabajo a lo largo de varios años. De esta manera, se proponen situaciones donde los alumnos en un ámbito de seminario, a partir de problemas sociales y tecnológicos construyan conocimiento ingenieril, incluyendo en él dimensiones éticas, políticas, económicas, culturales.

El desarrollo del proyecto por parte de los estudiantes dará lugar a que los mismos aborden situaciones que pueden resolverse según variados enfoques, propiciando de esta manera la estimulación del pensamiento creativo, el análisis crítico, el razonamiento y la iniciativa personal y comunitaria. Se sostiene que la tarea en grupo favorece la formación en el campo de las relaciones interpersonales, en el trabajo cooperativo y en las competencias comunicativas. Esta actividad grupal brindará oportunidades para el ejercicio de la comunicación, el intercambio de puntos de vista, la posibilidad de relación con diversas comunidades en una tarea de que requerirá tanto la generación de demandas como el acompañamiento en el uso del producto.

\section{REFERENCIAS}

Albizzati, E., A. Arese, D. Estenoz y G. Rossetti; Equipamiento para el Aprendizaje de los Fundamentos de la Transferencia de Cantidad de Movimiento, de Energía y de Materia, Información Tecnológica: 9 (4) , 349-355 (1998).

Albizzati, E.; Inclusión de Temas Relacionados con la Energía Solar en Cursos de las Carreras de Ingeniería, Proceeding ISES Millennium Solar Forum 2000, 17 al 22 de setiembre, 663-666, D.F. México (2000).

Albizzati, E. y G. Rossetti; Experiencia Didáctica de Calentamiento Aprovechando la Energía Solar, Revista Avances en Energías Renovables y Medio Ambiente, Vol.6, 10.37 - 10.42 (2002).

Arese, A. y otros cuatro autores; Estrategias de enseñanza: relaciones entre teoría $y$ experimentación, Memorias del 3er. Congreso Argentino de Enseñanza de Ingeniería, Bahía Blanca, 20 al 22 de setiembre, publicado en CD (2000).

Arese, A., M. del C. Castells, E. Albizzati y G. Rossetti; Elaboración de Estrategias de enseñanza en el marco de un proyecto de investigación- acción, Proceeding of VII International Conference on Engineering and Technology Education, http://www.asee.org/international/ intertech.cfm (2001). Acceso: 5 de mayo (2002)

Bauman, Z.; Vida de consumo, Ed. Fondo de Cultura Económica, Buenos Aires (2007).

Bourdieu, P.; El sentido práctico, Ed. Taurus, Madrid (1991).

Brennecke, J. y M. Stadtherr; A course in environmentally conscious chemical process engineering, Computers and Chemical Engineering: 26, 307-318 (2002).

Camilloni, A., D. Zadunaisky y M. Fernández; Estudios para la Reforma Curricular de la Universidad de Buenos Aires, Secretaría de Asuntos Académicos de la Universidad de Buenos Aires, Ed. Eudeba, Volumen 2, Buenos Aires (2002). 
Castells, M.; La ciudad de la nueva economía, Revista La factoría, 12 (2000). http://www.lafactoria web.com/ default-2.htm . Acceso 6 de noviembre (2006).

Castells, M. del C., N. Vega, A. Arese y S. Guala; Análisis de los planes de estudio en la Facultad de Ingeniería Química. Hacia una comprensión de la cultura institucional, Actas del "Congreso Latinoamericano de Ingeniería y Ciencias Aplicadas", ISBN 987-575-011-5, 2 al 4 de marzo de 2005 publicado en CD, San Rafael, Mendoza. (2005).

Charpentier, J.C.; The triplet "molecular process-product-process" engineering: the future of chemical engineering?, Chemical Engineering Science: 57, 4667-4690 (2002).

Chevallard, Y.; La transposición didáctica, Ed. Aique, Buenos Aires (1997).

Costa, R., G.D. Moggridge y P.M. Saraiva; Chemical Product Engineering: An emerging paradigm within Chemical Engineering, AIChE Journal: 52(6), 1976-1986 (2006).

Elliot, J.; El cambio educativo desde la investigación acción, Ed. Morata, Madrid (1993).

Gutiérrez Martin, F.; Nuevos Paradigmas y Métodos para la Educación Ambiental en la Ingeniería, Documento de las Jornadas de intercambio de Experiencias en Innovación Educativa en la UPM, Madrid, 6 al 8 de febrero de 2007,

http://www.upm.es/innovación/cd/09_cyj/documentos/intercambio_experiencias _innovacion.html. Acceso: 2 de noviembre (2007)

Morin, E.; Introducción al pensamiento complejo, Ed. Gedisa, Barcelona (1998).

Maciel de Oliveira, C.; La investigación-acción como estrategia de aprendizaje en la formación inicial del profesorado, Revista Iberoamericana de Educación, 33 (2003). http://www.rieoei.org/rie33a05 .htm. Acceso: 3 de julio (2006)

Monereo, C.; Estrategias de enseñanza y aprendizaje, 5 Edición, Ed. Graó, Barcelona (1998)

Najmanovich, D.; La complejidad: de los Paradigmas a las figuras del pensar, Revista Complexus: 1 (2), 67-76 (2005).

Osorio, C.M.; Los efectos de la ingeniería en el Aspecto Humano, Conferencia presentada en el XXIX Convención Panamericana de Ingeniería, UPADI 2004, Ciudad de México, 22 al 25 de octubre (2004).

Pereira, C.J.; Environmentally friendly processes, Chemical Engineering Science: 54, 1959-1973 (1999).

Pozo, J. y C. Monereo; El aprendizaje estratégico, Ed. Santillana S.A., Buenos Aires (2001).

Saraiva P. M., L.O. Santos y R.M. Quinta-Ferreira; Reengineering Chemical Engineering Education for the Future, Proceedings International Conference on Engineering Education - ICEE 2007, http://icee2007.dei.uc.pt/proceedings/papers/589.pdf. Acceso: 9 de noviembre (2007).

Stenhouse, L.; Investigación y desarrollo del currículo, Ed. Morata, Madrid (1991).

Tapias García, H.; Ingeniería Química: Escenario futuro y dos nuevos paradigmas, Rev. Ingeniería Química: julio-agosto, 179-186 (1999).

Wintermantel, K.; Process and product engineering - achievements, present and future challenges, Chemical Engineering Science: 54, 1601-1620 (1999). 